\title{
Successful olaparib desensitization using a novel one-day protocol
}

\author{
Rongbo Zhu ${ }^{1 *}\left(\mathbb{D}\right.$, Stephen Welch ${ }^{2}$ and Hannah Roberts ${ }^{1}$
}

\begin{abstract}
Background: Olaparib is a revolutionary treatment for patients with ovarian and breast cancer. Currently, there is no established 1-day drug desensitization protocol for patients with olaparib type-1 hypersensitivity reactions despite well documented IgE-mediated adverse reactions occurring with olaparib.

Case presentation: We report a 58-year-old female with immediate, reproducible IgE-mediated adverse reactions to olaparib tablets with implementation of a 1-day novel desensitization protocol to olaparib. Following desensitization, the patient was successfully transitioned from olaparib capsules to tablets with no loss of tolerance.

Conclusions: To our knowledge, this is the first reported case of successful olaparib desensitization using a novel 1-day desensitization protocol, and will contribute to drug allergy knowledge, in an area where robust data is lacking. This case demonstrates the important role for drug desensitization in patients with immediate hypersensitivity reactions to chemotherapeutic agents. Furthermore, as olaparib capsules are being phased out in favour of olaparib tablets, we provide a clear case that transitioning from capsule to tablet form did not cause a loss of tolerance.
\end{abstract}

Keywords: Olaparib, Drug desensitization, Drug allergy, Chemotherapy agent

\section{Background}

Cancer is a leading cause of death in Canada and has a major impact on the health care system [1]. Breast and ovarian cancer are the second and fifth leading cause of cancer related death among Canadian women [1]. Treatments for both cancers generally require aggressive multimodal interventions including surgery, cytotoxic chemotherapy, radiation and biologic therapy [2].

Olaparib is a poly (adenosine diphosphate (ADP)ribose) polymerase (PARP) inhibitor that is an effective maintenance therapy for patients with platinum sensitive, relapsed ovarian cancer and has provided significant progression-free survival [3]. Following its efficacy in ovarian cancer, it was approved as a novel agent for germline BRCA-mutated, HER2-negative, metastatic breast cancer [4] and very recently, it was approved in

*Correspondence: Rongbo.Zhu@lhsc.on.ca

1 Division of Clinical Immunology and Allergy, Department of Medicine, Western University, London, ON, Canada

Full list of author information is available at the end of the article
North America as a therapy for germline BRCA-mutated metastatic pancreatic cancer [5].

IgE-mediated adverse drug reactions to olaparib are rare, but have been reported [6, 7]. Drug desensitization is a safe and effective way to achieve temporary tolerance in individuals with IgE- mediated reactions to chemotherapy agents to ensure provision of optimal therapy $[8,9]$. There is only one report in the literature of successful olaparib desensitization, using a 2-day protocol with olaparib capsules [10]. Maintenance therapy on olaparib capsules (50 $\mathrm{mg}$ capsules) results in significant pill burden, as patients typically require 8 capsules twice daily (400 mg twice daily) [7]. In addition, olaparib capsules are being discontinued in some countries, favoring olaparib $150 \mathrm{mg}$ tablets [11].

We describe a patient with significant, reproducible IgE-mediated adverse reactions to olaparib tablets with implementation of a 1-day desensitization protocol to olaparib capsules, with subsequent successful transition from olaparib capsules to tablets. 


\section{Case presentation}

A 58-year-old female was referred to the allergy and clinical immunology service from medical oncology for evaluation of olaparib allergy. Her history is significant for recurrent high grade serous ovarian cancer with previous cytoreductive surgery, intraperitoneal chemotherapy and intravenous (IV) chemotherapy (paclitaxel and carboplatin). She was started on $300 \mathrm{mg}$ of olaparib (tablets) and $35 \mathrm{~min}$ after her first dose, developed extreme facial flushing and diffuse pruritus. She took diphenhydramine and proceeded with the second dose of olaparib $12 \mathrm{~h}$ later. This resulted in immediate lip and facial angioedema, along with pruritus. She administered diphenhydramine and cutaneous symptoms decreased in severity. She continued to take the third dose and within 30 min developed diffuse pruritus, facial urticaria, periorbital and lip angioedema, and nausea. Olaparib was discontinued. There were no symptoms suggestive of a delayed adverse drug reaction. She had no history of chronic spontaneous urticaria and angioedema. She proceeded to take diphenhydramine every $6 \mathrm{~h}$ and cutaneous symptoms resolved within $24 \mathrm{~h}$.

Given the patient's convincing history and reproducible immediate, IgE-mediated symptoms, a Type 1 hypersensitivity reaction to olaparib was diagnosed [8]. As per hospital regulations for the safe handling of cytotoxic medications, epicutaneous testing was not performed at our center. Skin testing was not deemed necessary based on a highly convincing clinical history with objective symptoms witnessed by physicians, nonstandardized olaparib skin testing, and the imminent need for olaparib as a first-line therapy.

The patient was admitted as an inpatient and successfully underwent a 9 step, 1-day desensitization protocol we developed (Table 1). As the standard treatment dose of olaparib is $400 \mathrm{mg}$ twice daily, we aimed for a cumulative dose in our desensitization to reflect that. She was pre-medicated with $20 \mathrm{mg}$ of oral cetirizine, $10 \mathrm{mg}$ of montelukast, $50 \mathrm{mg}$ of IV ranitidine, and $10 \mathrm{mg}$ of IV dexamethasone prior to desensitization. During the desensitization, she developed several isolated erythematous macules, but no urticaria, angioedema, or any systemic involvement at a cumulative dose of $450 \mathrm{mg}$ of olaparib capsules. With close monitoring, the desensitization continued to completion with no interruptions and the macules resolved with no intervention required.

The patient successfully continued on oral olaparib capsules with no further adverse reactions. However, given the pill burden of olaparib capsules, she was seen in follow-up as an outpatient 3 weeks later, where she underwent a supervised ingestion to olaparib tablets (Table 2), with no immediate or delayed adverse reaction. The patient has since continued on olaparib tablets without issue and has been monitored in follow-up.

\section{Discussion and conclusions}

We report the first case of one-day desensitization for an IgE-mediated allergy to olaparib. To our knowledge, there is only one other report of successful olaparib desensitization, using a 2-day protocol with olaparib capsules [5]. One-day drug desensitization protocols are preferred over multiple day protocols as they decrease hospital resources and are more convenient for both the patient and the medical team [9].

Table 2 Supervised ingestion and transition to olaparib tablets

\begin{tabular}{lllll}
\hline Steps & $\begin{array}{l}\text { Olaparib } \\
\text { tablet dose } \\
(\mathbf{m g})\end{array}$ & $\begin{array}{l}\text { Cumulative } \\
\text { dose (mg) }\end{array}$ & $\begin{array}{l}\text { Interval } \\
\text { since previous } \\
\text { dose (minute) }\end{array}$ & $\begin{array}{l}\text { Cumulative } \\
\text { duration } \\
\text { (Hour:Minute) }\end{array}$ \\
\hline 1 & 150 & 150 & - & $00: 00$ \\
2 & 150 & 300 & 30 & $00: 30$ \\
\hline
\end{tabular}

Table 1 1-day olaparib desensitization protocol

\begin{tabular}{|c|c|c|c|c|}
\hline Steps & Olaparib capsule dose $(\mathrm{mg})$ & Cumulative dose (mg) & $\begin{array}{l}\text { Interval since previous dose } \\
\text { (minute) }\end{array}$ & $\begin{array}{l}\text { Cumulative } \\
\text { duration } \\
\text { (Hour:Minute) }\end{array}$ \\
\hline 1 & 50 & 50 & - & $00: 00$ \\
\hline 2 & 50 & 100 & 30 & $00: 30$ \\
\hline 3 & 50 & 150 & 30 & 01:00 \\
\hline 4 & 100 & 250 & 60 & 02:00 \\
\hline 5 & 100 & 350 & 60 & 03:00 \\
\hline 6 & 100 & 450 & 90 & $04: 30$ \\
\hline 7 & 150 & 600 & 90 & 06:00 \\
\hline 8 & 150 & 750 & 120 & 08:00 \\
\hline 9 & 150 & 900 & 120 & $10: 00$ \\
\hline
\end{tabular}


There is currently no literature on transitioning from olaparib capsules to tablets after desensitization. Our case is the first to provide evidence that it is safe to transition from olaparib capsules to tablets after desensitization with maintenance of drug tolerance. This becomes increasingly important as olaparib capsules continue to be phased out in favour of olaparib tablets [11].

Our case demonstrates that one-day drug desensitization for olaparib is safe and exemplifies the important role for drug desensitization in patients with immediate hypersensitivity reactions to chemotherapeutic agents. To our knowledge, this is the first reported case of successful olaparib desensitization using a novel 1-day desensitization protocol, and will contribute to drug allergy knowledge, especially chemotherapeutic adverse reactions, in an area where robust data is lacking.

\section{Abbreviations}

ADP: Adenosine diphosphate; BRCA: Breast cancer gene; HER2: Human epidermal growth factor receptor 2; IgE: Immunoglobulin E; IV: Intravenous; PARP: Poly-ADP-ribose polymerase.

\section{Acknowledgements}

We would like to acknowledge nursing support during desensitization.

\section{Authors' contributions}

$\mathrm{RZ}$, SW and HR were involved in the conception and writing of the manuscript. All authors read and approved the final manuscript.

\section{Funding}

No funding was received for this work.

\section{Availability of data and materials}

All data generated or analyzed during this study are included in this published article.

\section{Ethics approval and consent to participate}

Not applicable.

\section{Consent for publication}

Informed consent was obtained from patient for publication.

\section{Competing interests}

SW has been involved in clinical trials and received honoraria from AstraZeneca.

\section{Author details}

${ }^{1}$ Division of Clinical Immunology and Allergy, Department of Medicine, Western University, London, ON, Canada. ${ }^{2}$ Division of Medical Oncology, Department of Oncology, Western University, London, ON, Canada.
Received: 8 May 2020 Accepted: 2 November 2020

Published online: 11 November 2020

\section{References}

1. Brenner DR, Weir HK, Demers AA, Ellison LF, Louzado C, Shaw A, et al. Projected estimates of cancer in Canada in 2020. CMAJ. 2020;192(9):E199-205.

2. Arruebo M, Vilaboa N, Sáez-Gutierrez B, Lambea J, Tres A, Valladares M, et al. Assessment of the evolution of cancer treatment therapies. Vol. 3, Cancers. Multidisciplinary Digital Publishing Institute (MDPI); 2011. p. 3279-330.

3. Pujade-Lauraine E, Ledermann JA, Selle F, Gebski V, Penson RT, Oza AM, et al. Olaparib tablets as maintenance therapy in patients with platinumsensitive, relapsed ovarian cancer and a BRCA1/2 mutation (SOLO2/ ENGOT-Ov21): a double-blind, randomized, placebo-controlled, phase 3 trial. Lancet Oncol. 2017;18(9):1274-84.

4. Robson M, Im SA, Senkus E, Xu B, Domchek SM, Masuda N, et al. Olaparib for metastatic breast cancer in patients with a germline BRCA mutation. N Engl J Med. 2017;377(6):523-33.

5. Golan T, Hammel P, Reni M, Van Cutsem E, Macarulla T, Hall MJ, et al. Maintenance olaparib for germline BRCA-mutated metastatic pancreatic cancer. N Engl J Med. 2019;381(4):317-27.

6. LaFargue CJ, Dal Molin GZ, Sood AK, Coleman RL. Exploring and comparing adverse events between PARP inhibitors. Vol. 20, The Lancet Oncology. Lancet Publishing Group; 2019. p. e15-28.

7. Canada A. PRODUCT MONOGRAPH INCLUDING PATIENT MEDICATION INFORMATION LYNPARZA ${ }^{\circledR}$ olaparib capsules 50 mg Antineoplastic agent [Internet]. 2020

8. Solensky R, Khan DA, Contributors Leonard Bernstein WI, Bloomberg GR, Castells MC, Mendelson LM, et al. Drug allergy: An updated practice parameter. Ann Allergy, Asthma Immunol. 2010;105(4).

9. Sloane D, Govindarajulu U, Harrow-Mortelliti J, Barry W, Hsu FI, Hong D, et al. Safety, Costs, and Efficacy of Rapid Drug Desensitizations to Chemotherapy and Monoclonal Antibodies. J Allergy Clin Immunol Pract. 2016:4(3):497-504

10. Grabowski JP, Sehouli J, Glajzer J, Worm M, Zuberbier T, Fluhr JW. Olaparib desensitization in a patient with recurrent peritoneal cancer. Vol. 379, New England Journal of Medicine. Massachusetts Medical Society; 2018. p. $2176-7$.

11. Food and Drug Administration. FDA approves of olaparib tablets for maintenance treatment in ovarian cancer [Internet]. 2017. https://www. fda.gov/drugs/resources-information-approved-drugs/fda-approvesolaparib-tablets-maintenance-treatment-ovarian-cancer.

\section{Publisher's Note}

Springer Nature remains neutral with regard to jurisdictional claims in published maps and institutional affiliations.
Ready to submit your research? Choose BMC and benefit from:

- fast, convenient online submission

- thorough peer review by experienced researchers in your field

- rapid publication on acceptance

- support for research data, including large and complex data types

- gold Open Access which fosters wider collaboration and increased citations

- maximum visibility for your research: over 100M website views per year

At BMC, research is always in progress.

Learn more biomedcentral.com/submissions 